\title{
Percutaneous renal artery denervation in patients with chronic systolic heart failure: A randomized controlled trial
}

\author{
Jun-Qing Gao, Wei Yang, Zong-Jun Liu \\ Department of Cardiology, Putuo Hospital, Shanghai University of \\ Traditional Chinese Medicine, Shanghai, People's Republic of China
}

\begin{abstract}
Background: Renal denervation (RDN) is as an effective treatment for heart failure (HF), but its effects on cardiac function of patients with HF are not well documented. Here, the aim was to investigate RDN's effect on patients with chronic systolic HF, by conducting a single-center, prospective, randomized, and controlled study.

Methods: Sixty patients with chronic systolic HF were randomly assigned to the RDN or control groups, receiving percutaneous catheter-based RDN with radiofrequency ablation and drug treatment, respectively. All patients performed a 6-minute walk test, echocardiography, blood pressure measurement, and biochemical test, at both baseline and in a 6-month follow up.

Results: Over 6-month follow up, patients in RDN group showed a decrease in $N$-terminal pro-B-type natriuretic peptide $(440.1 \pm 226.5 \mathrm{pg} / \mathrm{mL}$ vs. $790.8 \pm 287.0 \mathrm{pg} / \mathrm{mL}, p<0.001$, Cohen's $d=1.14)$, an increase in left ventricular ejection fraction $(39.1 \pm 7.3 \%$ vs. $35.6 \pm 3.3 \%, p=0.017$, Cohen's $d=0.61)$, improved New York Heart Association class assessment ( $p=0.01$, Cohen's $d=0.66)$, and decreased blood pressures $(p<0.001$, Cohen's $d=0.91)$, without reporting hypotension and syncope amaurosis. No significant between-group difference was observed for glomerular filtration rate and heart rate.

Conclusions: Renal denervation which effectively and safely improves patient's cardiac function as well as exercise tolerance, could be considered as an effective treatment for chronic systolic HF. (Cardiol J 2019; 26, 5: 503-510)
\end{abstract}

Key words: renal denervation, sympathetic nervous system, heart failure, blood pressure, cardiology

\section{Introduction}

Chronic heart failure (HF) is a common disease suffered by around 100 million people all over the world. There were 5.1 million people in United States at present a number which increases at a rate of 800,000 cases per year whosuffer from this disease. HF is believed to be a major cause of sudden cardiac death, and previous studies have shown that the death rate for patients with chronic $\mathrm{HF}$ is $6-9$ times higher than those in the normal population. Around 300,000 deaths are caused by HF every year in United States [1]. The medical expense on $\mathrm{HF}$ reachedas much as $\$ 30$ billion in 2012, and has kept growing in recent years [2].

Excessive activity of the sympatheic nerve system is believed as one of the major causes of the HF progression. Sympathetic activation involves efferent and afferent pathways to regulate cardiovascular functions such as blood pressure $(\mathrm{BP})$ and heart rate, in response to acute stress like volume depletion or excessive vasodilatation [3]. Exces-

Address for correspondence: Dr. Zong-Jun Liu, Department of Cardiology, Putuo Hospital, Shanghai University of Traditional Chinese Medicine, Shanghai 200062, People's Republic of China, tel: 021-22233222, fax: 021-52665957, e-mail: 1zj160507@163.com

Received: 2.09.2017 Accepted: 15.01.2018 
sive activity of the sympathetic nervous system has a direct adverse consequence for cardiovascular diseases, particularly for HF and hypertension [4]. A proper suppression of the sympathetic nervous system activity may be able to improve the conditions of patients with HF. Particularly, catheterbased renal sympathetic denervation (RDN), which can specificly and effectively reduce the activity of sympatheic nervous system [3,5], which is considered a proper treatment for HF.

Renal denervation was implemented by selective sympathetic denervation of the human kidney with radiofrequency energy ablation. It was firstly proposed to treat resistant hypertension $[6,7]$, but its treatment effect for this specific disease was not as good as had been imagined. HTN-3 study found that there was no significant difference in BP between the RDN and sham groups [8]. Even though, its ability in suppressing the activity of the sympathetic nervous system suggests it is a possible application in the treatment of HF.

To explore the treatment effect of RDN on HF, several animal models, e.g.pig and rat models, were established [9-11] and the performance of RDN on these animal models were quite promissing. However, these animal based results were insufficient to be considered as direct evidence for the application of RDN in clinical practice; more experiments on humans should be conducted to eliminate the concerns regarding its effectiveness and safety to patients with HF. A first-in-man study of chronic systolic HF has already been conducted [12], and suggests that patient exercise tolerances were improved after the intervention of RDN. Nevertheless, small sample size and its single-group non-blinded and non-randomized nature limited the validity of these research findings. Thus, the aim presently was to comprehensively investigate the effectiveness and safety of catheter-based $\mathrm{RDN}$ in the treatment of chronic systolic HF, by conducting a randomized controlled trial with a larger sample size.

\section{Methods}

\section{Patients}

Sixty patients with chronic systolic HF (New York Heart Association [NYHA] class II or III) were recruited in this study. All patients were not less than 18 years old. The inclusion criteria were: (1) left ventricular ejection fraction (LVEF) should be smaller than $40 \%$ at echocardiography or N-terminal pro-B-type natriuretic peptide (NT-proBNP) should be larger than $125 \mathrm{pg} / \mathrm{mL}$; (2) glomerular filtration rate (GFR) should not be smaller than $45 \mathrm{~mL} / \mathrm{min} / 1.73 \mathrm{~m}^{2}$ and systolic BP should not be smaller than $100 \mathrm{mmHg}$. The exclusion criteria were: (1) patients with renal artery stenosis (in history or revealed by imaging), type I diabetes, severe heart valvar disease, and myocardial infarction or cerebrovascular accident 6 months prior were excluded; (2) patients who were or would have been in pregnancy during the study.

The study was approved by the ethics committee of Putuo Hospital Affiliated to Shanghai University of Traditional Chinese Medicine. All patients had been informed in advance and signed a consent form.

\section{Experimental design}

This was a single-centre, prospective, randomized and controlled study. The 60 recruited patients were equally and randomly assigned to the intervention group undergoing catheter-based $\mathrm{RDN}$ and the control group receiving drug treatment with the random envelope method, there were 30 patients in each group. All involved patients were followed up for 6 months.

\section{RDN intervention}

Before the RDN intervention, patients were given enteric-coated acetylsalicylic acid (300 $\mathrm{mg}$ ) or clopidogrel (300 mg) by chewing. During the operation, patients were given heparin (6000-8000 U) by intravenous injection. Femoral artery puncture were conducted after skin preparation in right fold inguen and disinfection; then $7 \mathrm{~F}$ vascular sheath was imbedded. Renal arteriography was conducted in both left and right with a JR4 catheter. Spiral ablation was performed with an imbedded $6 \mathrm{~F}$ radiofrequency ablation catheter (Ablation instrument [39D-72X]: Johnson Medical Instrument Co. Ltd.) in temperature control mode $\left(8-10 \mathrm{~W}, 50^{\circ} \mathrm{C}\right)$ to both left and right renal arteries. There were $4-6$ ablation points in the left and right renal arteries respectively. The effective ablation time for each point was $60 \mathrm{~s}$ and the interval between two neighbouring points was $0.5 \mathrm{~cm}$. Renal arteriography was conducted after operation.

\section{Study assessment}

Efficacy endpoint. All patients accepted NT-proBNP test, echocardiographic LVEF assessment and NYHA class assessment both before the operation and after 6-month follow up. Six-minute walk test was also conducted both preoperatively and monthly during the following period. Changes in these tests were treated as the efficacy endpoint in this study. 
Safety endpoint. All patients accepted the office BP measurement both preoperatively and monthly during the follow up. Heart rate and GFR were also measured both before the operation and after 6-month follow up. Changes in these measurements were treated as safety points in this study.

\section{Pharmacological therapies}

All patients underwent pharmacotherapy for $\mathrm{HF}$ with a maximal tolerated dose prior to denervation, including beta-blockers, angiotensin converting enzyme (ACE) inhibitors or endothelin receptor antagonist, and spironolactone. No change of medications was permitted prior to denervation. During the following, patients were given standard $\mathrm{HF}$ care and the physicians could freely adjust the dosage of pharmacotherapy according to patient condition, e.g., changes in $\mathrm{BP}$ and heart rate.

\section{Statistical analysis}

Statistical analyses were carried out with the SPSS 21.0 statistical analysis package (SPSS Inc., New York, USA). All measurement data were presented as mean \pm standard deviation (SD); while, the enumeration data were presented as a percentage number. The comparison of measurement data was achieved by the student independent t-test with a prior checking of dataset normality by Shapiro-Wilk test; and for enumeration data, they were compared by $\chi^{2}$ test or the Fisher exact test. Statistical difference was defined as the situation where $\mathrm{p}$-value was smaller than 0.05 .

\section{Results}

\section{Baseline clinical characteristics}

Sixty patients ( 13 females and 47 males) with a mean age of $60.2 \pm 11.6$ years were enrolled in this randomized controlled study between January 2014 and July 2015. Their mean body mass index was $26.7 \pm 2.7 \mathrm{~kg} / \mathrm{m}^{2}$. Among these patients, $65.0 \%$ were with hypertension, $58.3 \%$ were with coronal heart disease, $11.7 \%$ were with atrial fibrillation, and $25 \%$ were with type 2 diabetes. All patients were diagnosed as chronic HF (NYHA class II or III, NT-proBNP level is $791.2 \pm 363.7 \mathrm{pg} / \mathrm{mL}$, 6 -minute walk distance is $213.8 \pm 65.9 \mathrm{~m}$, and the LVEF is $34.9 \pm 3.2 \%$ during the echocardiogram). There was a mean GFR of $100.6 \pm 33.9 \mathrm{~mL} /$ $/ \mathrm{min} / 1.73 \mathrm{~m}^{2}$, mean office systolic BP was $142.6 \pm$ $\pm 22.6 \mathrm{mmHg}$, mean diastolic $\mathrm{BP}$ was $80.8 \pm 12.6$ $\mathrm{mmHg}$, and mean heart rate was $69.1 \pm 7.3 \mathrm{bpm}$. In addition, $95 \%$ of patients were treated with
ACE inhibitors or angiotensin II receptor blockers, $78.3 \%$ with beta-blockers, $16.7 \%$ with aldosterone antagonists, $43.3 \%$ with calcium-channel blockers, $6.7 \%$ with digoxin, and $50 \%$ with loop diuretics.

Bilateral renal denervation was successfully performed in all 30 patients in the RDN group. No statistically significant difference was found between the two groups in age, sex, most comorbidities, reported duration spent on pharmacotherapy for $\mathrm{HF}$, and assessments of effectiveness and safety endpoints (Table 1).

\section{Efficacy endpoint}

All patients in both groups were followed up for 6 months. Results of the Student independent t-test on NT-proBNP indicates that the NT-proBNP levels in RDN group $(440.1 \pm 226.5 \mathrm{pg} / \mathrm{mL})$ were significantly lower ( $<<0.001$, Cohen's $d=1.14$ ) than those in the control group $(790.8 \pm 287.0 \mathrm{pg} /$ $/ \mathrm{mL}$ ) in the $6^{\text {th }}$ month follow up (Fig. 1).

The effects of RDN on cardiac functions of patients with chronic HF can be indicated by the results of independent t-tests on echocardiographic parameters between the two groups (Table 2). It is seen that LVEF significantly increased ( $\mathrm{p}=0.017$, Cohen's $d=0.61$ ) in the RDN group $(39.1 \pm 7.3 \%)$ when compared with the control group $(35.6 \pm 3.3 \%)$ at the end of the 6 -month follow up. Further, left ventricular end systolic diameters in the RDN group $(46.4 \pm 4.7 \mathrm{~mm})$ were significantly smaller ( $<0.001$, Cohen's $d=0.87$ ) than those in the control group $(50.2 \pm 3.1 \mathrm{~mm})$; nevertheless, no statistically significant difference was found in left ventricular end diastolic diameter and interventricular septal thickness between the two groups $(\mathrm{p}>0.05)$.

After 6-month follow up, patients in the RDN group showed an improvement of cardiac function, with 7 patients showing better results of NYHA class assessment (Fig. 2). The 6-minute walk distance in the RDN group $(301.2 \pm 139.5 \mathrm{~m})$ was significantly increased ( $\mathrm{p}=0.01$, Cohen's $d=0.66$ ) when compared with the control group $(227.2 \pm$ $\pm 65.0 \mathrm{~m}$ ) in the $6^{\text {th }}$ month (Fig. 3 ).

\section{Safety endpoint}

In the $6^{\text {th }}$ month, the office $\mathrm{BP}$ of patients in the RDN group $(123.3 \pm 0.9 / 68.5 \pm 7.0 \mathrm{mmHg})$ significantly decreased ( $\mathrm{p}<0.001$, Cohen's $d=0.91$ ) when compared with those in the control group $(139.8 \pm 20.7 / 80.2 \pm 11.8 \mathrm{mmHg})$, as shown in Figure 4; nevertheless, no patient reported symptoms of hypotension syncope or amaurosis during the follow up period. No significant differ- 
Table 1. Baseline patient characteristics, demographics, background medications, efficacy and safety endpoints parameters in renal denervation and control groups.

\begin{tabular}{|c|c|c|c|}
\hline & $\begin{array}{l}\text { Renal denervation group } \\
\qquad(\mathrm{n}=30)\end{array}$ & $\begin{array}{l}\text { Control group } \\
(\mathbf{n}=30)\end{array}$ & $\mathbf{P}$ \\
\hline Age [years] & $59.0(12.1)$ & $61.3(11.1)$ & 0.446 \\
\hline Sex [male] & $25(83.3 \%)$ & $22(73 \%)$ & 0.347 \\
\hline Body mass index $\left[\mathrm{kg} / \mathrm{m}^{2}\right]$ & $26.8(2.6)$ & $26.7(2.8)$ & 0.939 \\
\hline \multicolumn{4}{|l|}{ Medical history: } \\
\hline Hypertension & $21(70.0 \%)$ & $18(60.0 \%)$ & 0.417 \\
\hline Coronary heart disease & $17(56.7 \%)$ & $18(60.0 \%)$ & 0.793 \\
\hline Atrial fibrillation & $4(13.3 \%)$ & $3(10.0 \%)$ & 0.688 \\
\hline Type 2 diabetes & $8(26.7 \%)$ & $7(23.3 \%)$ & 0.766 \\
\hline \multicolumn{4}{|l|}{ Patients receiving drug class: } \\
\hline ACE or ARB & $29(96.7 \%)$ & $28(93.3 \%)$ & 1.000 \\
\hline Beta-blockers & $23(76.7 \%)$ & $24(80.0 \%)$ & 0.754 \\
\hline Aldosterone antagonists & $6(20.0 \%)$ & $4(13.3 \%)$ & 0.488 \\
\hline Calcium-channel blockers & $14(46.7 \%)$ & $12(40.0 \%)$ & 0.602 \\
\hline Digoxin & $3(10 \%)$ & $1(3.3 \%)$ & 0.612 \\
\hline Loop diuretics & $14(46.7 \%)$ & $16(53.3 \%)$ & 0.606 \\
\hline Hemoglobin & $119.1(25.9)$ & $112.3(24.5)$ & 0.299 \\
\hline \multicolumn{4}{|l|}{ Efficacy endpoint: } \\
\hline NT-proBNP [pg/mL] & $797.7(356.1)$ & $784.7(377.1)$ & 0.892 \\
\hline Six minute walk test [m] & $217.5(69.5)$ & $210.0(63.0)$ & 0.666 \\
\hline \multicolumn{4}{|l|}{ NYHA class: } \\
\hline Class II & $17(56.7 \%)$ & $16(53.3 \%)$ & 0.795 \\
\hline Class III & $13(43.3 \%)$ & $14(46.7 \%)$ & 0.795 \\
\hline Echocardiographic LVEF [\%] & $35.0(3.2)$ & $34.8(3.2)$ & 0.872 \\
\hline \multicolumn{4}{|l|}{ Safety endpoint: } \\
\hline GFR $\left[\mathrm{mL} / \mathrm{min} / 1.73 \mathrm{~m}^{2}\right]$ & $104.8(35.9)$ & $96.4(31.8)$ & 0.337 \\
\hline Symbolic BP [mmHg] & $142.0(24.8)$ & $143.2(20.7)$ & 0.844 \\
\hline Diastolic BP [mmHg] & $79.8(12.6)$ & $81.7(12.9)$ & 0.579 \\
\hline Heart rate $[\mathrm{bpm}]$ & $68.7(7.9)$ & $69.4(6.9)$ & 0.728 \\
\hline
\end{tabular}

Data are mean (standard deviation) or number (\%). ACE — angiotensin-converting enzyme inhibitor; ARB - angiotensin II receptor blocker; $\mathrm{BP}$ - blood pressure; GFR - glomerular filtration rate; LVEF — left ventricular ejection fraction; NT-proBNP — N-terminal pro-B-type natriuretic peptide; NYHA - New York Heart Association

ence was found for heart rates $(\mathrm{p}=0.062$, Cohen's $d=0.49)$ and GFR $(\mathrm{p}=0.209$, Cohen's $d=0.33)$ between the two groups after 6 -month follow up, even though there was a slight decrease in heart rate and an increase in GFR were observed (Figs. 5, 6).

\section{Discussion}

This was a prospective randomized and controlled study to explore the effectiveness and safety of cathedral-based RDN on patients with chronic systolic HF. The improvement of exercise tolerance, decrease of NT-proBNP level, and increase of LVEF at echocardiography, suggest the feasibility and effectiveness of RDN in treatment of chronic systolic HF. In addition, no amaurosis and syncope in the RDN group, as well as no significant difference between-group of GFR further indicate the safety of RDN treatment.

Renal denervation has never been proposed as an effective way to treat resistant hypertension $[6,7]$, but its effect on this specific disease was inconclusive due to disappointing results acquired in the SIMPLICITY HTN-3 study, no significant difference was found in reduced BP between the RDN and sham groups [8]. Even though, recent studies proposed that RDN might be an effective way for the treatment of HF [12], due to its abilities in 


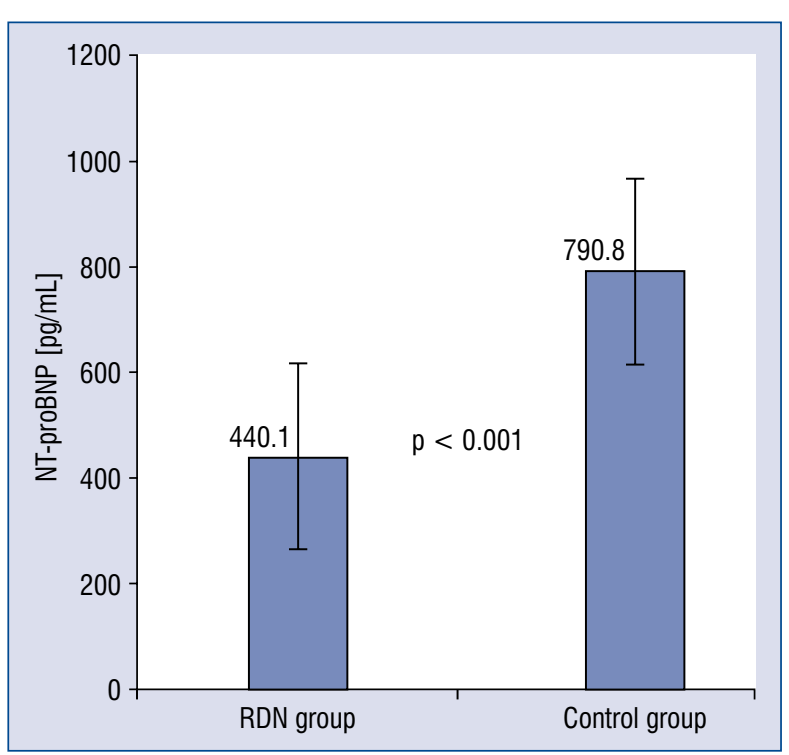

Figure 1. Mean N-terminal pro B-type natriuretic peptide (NT-proBNP) level at 6 months after renal denervation (RDN) in the RDN and control groups. A significant decrease in the RDN group was observed. Error bars indicate standard errors.

suppressing activities of the sympathetic nervous system and renin-angiotensin-aldosterone system (RASS) by blocking the renal sympathetic nerve. It should be noted that excessive activation of the sympathetic nerves and the over expression of RASS are believed to be major contributors to the cause and development of HF [13]. Actually, excessive activation of the renal sympathetic nerves can lead to renal vasoconstriction and increase renin secretion as well as proximal tubular sodium reabsorption [14]. This kind of chronic stimulation caused by excessive activation of the sympathetic nerves can lead to volume overloading, myocardial remodeling and cardiovascular function deterioration [15].

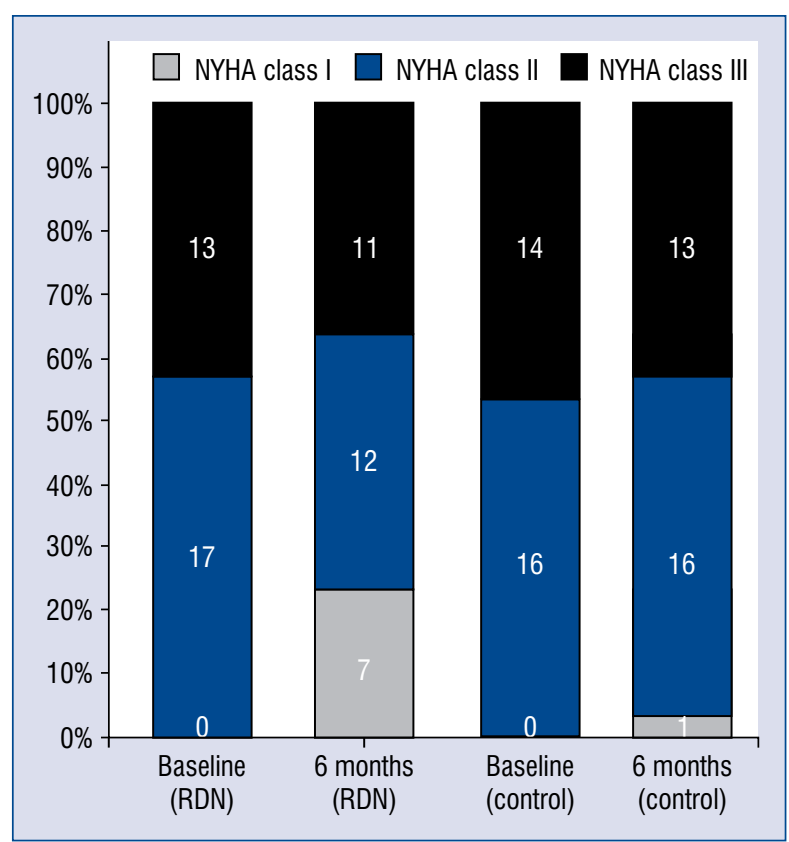

Figure 2. New York Heart Association (NYHA) class assessment in the renal denervation (RDN) and control groups at baseline and at 6 months after RDN. Black bars indicate NYHA class III. Blue bars indicate NYHA class II. Gray bars indicate NYHA class I.

Accumulating animal evidence suggests that $\mathrm{RDN}$ is able to bring hemodynamic changes and improve cardiac functions. For example, RDN showed an effective inhibition on RASS in a porcine model of pacing-induced HF [11]. After pacing, the RDN group has shown a significantly higher LVEF at echocardiography and a significantly lower plasma concentration of renin, relative to the control group. In contrast, although a significant elevated plasma concentration of aldosterone was reported in the control group, no significant change in the aldosterone level was observed in the RDN group. Similar results have been found in a rat model of

Table 2. Changes in echocardiographic parameters before and 6 months after randomization in the renal denervation (RDN) and control groups.

\begin{tabular}{|c|c|c|c|c|c|c|}
\hline \multirow[t]{2}{*}{ Parameter } & \multicolumn{3}{|c|}{ Baseline } & \multicolumn{3}{|c|}{6 months } \\
\hline & RDN & Control & $\mathbf{P}$ & RDN & Control & $\mathbf{P}$ \\
\hline LVEF [\%] & $35.0 \pm 3.2$ & $34.8 \pm 3.2$ & 0.872 & $39.1 \pm 7.3$ & $35.6 \pm 3.3$ & 0.017 \\
\hline LVESD [mm] & $50.3 \pm 3.3$ & $51.4 \pm 2.4$ & 0.156 & $46.4 \pm 4.7$ & $50.2 \pm 3.1$ & $<0.001$ \\
\hline LVEDD [mm] & $63.3 \pm 4.5$ & $63.6 \pm 4.7$ & 0.082 & $60.0 \pm 6.7$ & $62.5 \pm 4.5$ & 0.106 \\
\hline IVST [mm] & $10.3 \pm 1.3$ & $10.2 \pm 1.3$ & 0.848 & $9.5 \pm 0.9$ & $9.6 \pm 0.9$ & 0.575 \\
\hline
\end{tabular}

Data are mean \pm standard deviation. LVEF — left ventricular ejection fraction; LVESD — left ventricular end systolic diameter; LVEDD — left ventricular end diastolic diameter; IVST — interventricular septal thickness 


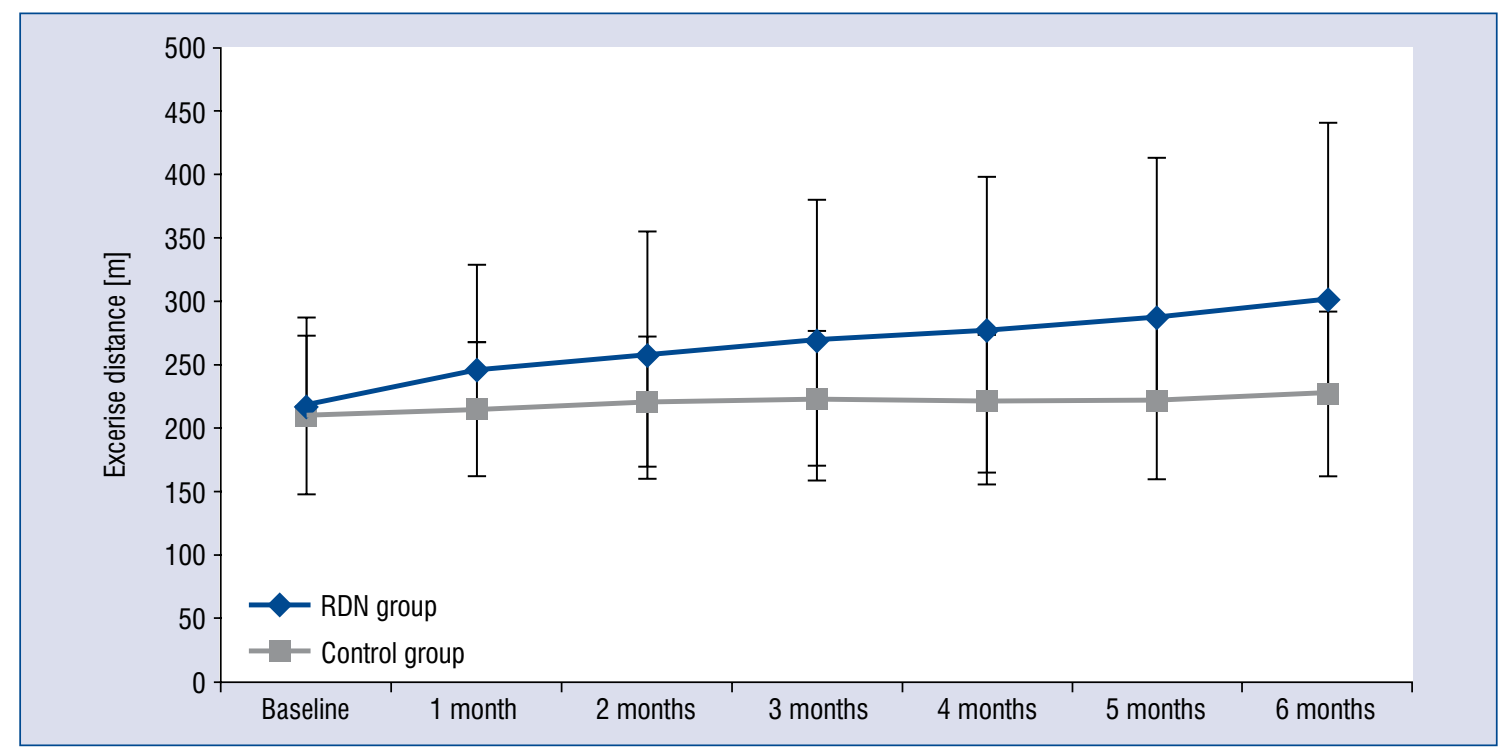

Figure 3. Change in 6 minute walk test before and after renal denervation (RDN) for RDN and control groups. A 6 minute walk test was measured at baseline and over a 6 month follow-up. A significant improvement in 6 minute walking distance was observed in the RDN group over 6 months. Error bars indicate standard errors.

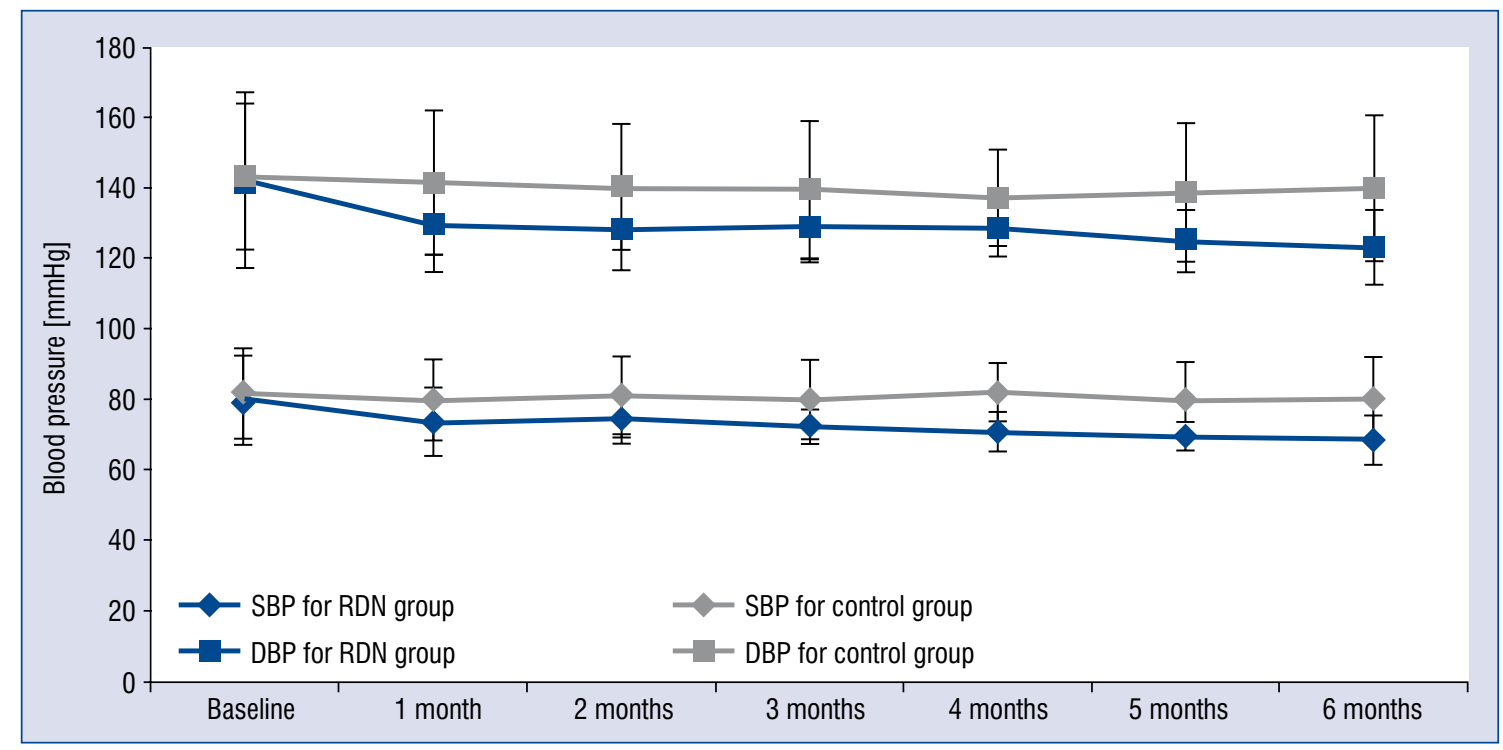

Figure 4. Paired changes of systolic blood pressure (SBP) and diastolic blood pressure (DBP) before and after renal denervation (RDN) for RDN and control groups. Blood pressure was measured at baseline and over a 6 month followup. Significant reductions in blood pressure were observed in the RDN group over 6 months. Error bars indicate standard errors.

isoproterenol induced chronic HF. It was reported that RDN down-regulated the protein expression of angiotensin II in myocardial tissue of rats left atrial, and thereby improved the cardiac function, shown by an increased LVEF [10]. Moreover, RDN was reported to be able to improve the cardiac function and inhibit myocardial remodeling in rats with post-myocardial infarction [9]. Further animal study has suggested that denervation intervention can ameliorate progression of left ventricular hypertrophy in spontaneously hypertensive rats, probably due to the reduction of $\mathrm{BP}$ and lower expression of inflammatory factors (e.g., TLR4, NF- $\kappa \mathrm{B}, \mathrm{TNF}-\alpha$, and IL-6) in myocardial tissue [16]. 


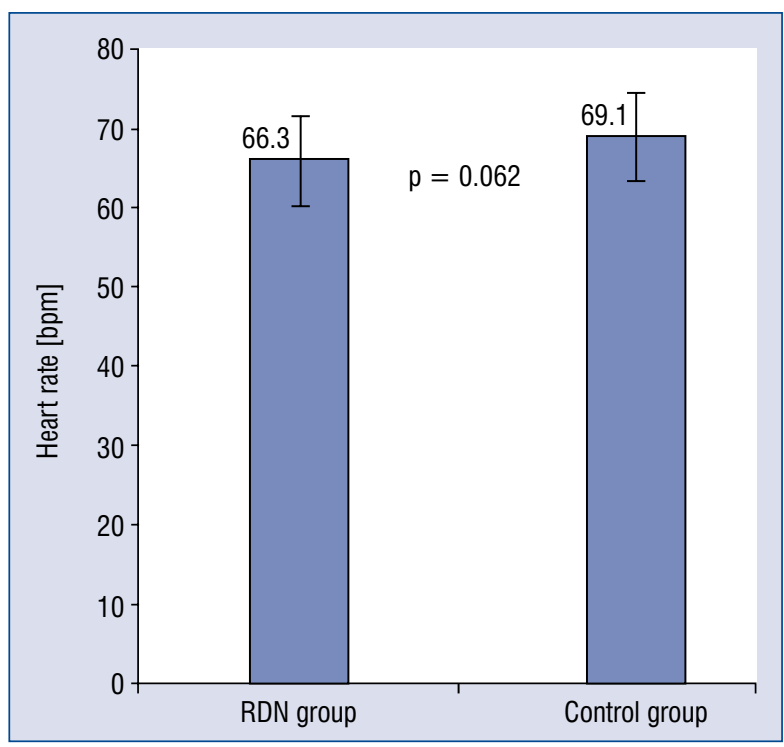

Figure 5. Mean heart rate at 6 months after renal denervation (RDN) in the RDN and control groups. No significant between-group differences were observed. Error bars indicate standard errors.

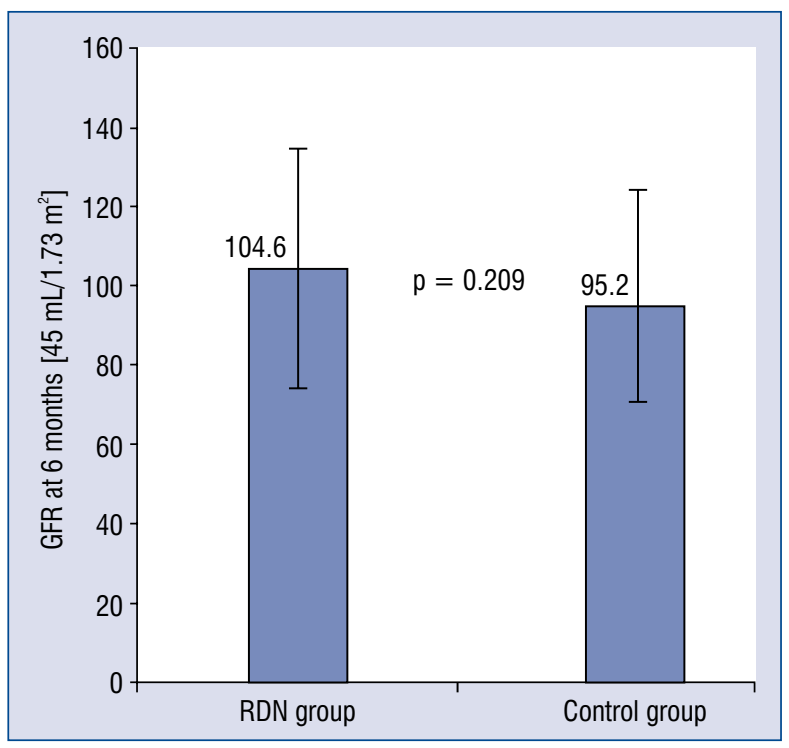

Figure 6. Mean glomerular filtration rate (GFR) at 6 months after renal denervation (RDN) in the RDN and control groups. No significant between-group differences were observed. Error bars indicate standard errors.

Though the positive results have been well confirmed in animal studies, the effects of RDN on patients with $\mathrm{HF}$ are still not well documented, and some indirect evidence were reported in studies on treatment of resistant hypertension. For example, a study including 72 patients reported that RDN could ameliorate the cardiac function, indicated by an improvement in left ventricular mass index, left ventricular mass/body surface area ratio, left ventricular wall stress, as well as LVEF values [17]. Another study also reported that the resistant hypertension patients were also accompanied with left ventricular hypertrophy and diastolic dysfunction [18]. These results together suggest that RDN would be promising treatment for $\mathrm{HF}$, especially for that accompanied with hypertension. Actually, the REACH-Pilot study has once investigated the effects of RDN on patients with $\mathrm{HF}$ and reported that RDN could improve patient exercise tolerances [12]. Nevertheless, this study was limited by its small sample size and its single-group non-blinded and non-randomized nature [19]. Here, more patients were recruited and a randomized controlled trial was performed for more convincing results. Obtained results verfied the values of RDN in improving the cardiac function and treating patients with $\mathrm{HF}$.

The RDN group in this study had shown a reduction in $\mathrm{BP}$ of approximately $20 \mathrm{mmHg}$ over 6-month follow up. However, such changes in BP did not accompany any symptoms of hypotension, indicating that this intervention did not lead to symphonic problems in patients with chronic systolic HF. Furthermore, patients receiving RDN showed no changes in GFR and heart rate before and after the procedures. The reduction of $\mathrm{BP}$ has been suggested to potentially dampen the impairment of renal function, as a decreased BP may help reduce the sympathetic outflow to the kidney [6]. In this study, the hemodynamic changes and normal renal function show no adverse effects on the kidneys of patients with chronic systolic BP, suggesting the safety of RDN.

\section{Limitations of the study}

Several limitations need to be noted in this study. First, the number of patients with systolic $\mathrm{HF}$ for RDN procedure was not large, thereby limiting statistical power. However, the improvement of cardiac function in the present population is in line with that of previous studies in hypertension with a large sample size. Second, non-blinded or sham-procedure group was not provided. The results, therefore, may contain bias. A randomized controlled trial with a sham group should be done to further investigate the effectiveness of RDN in HF. Third, whether RDN treatment in chronic systolic HF could improve a patients' diuretic need or resistance warrants further investigation. 


\section{Conclusions}

Catheter-based $\mathrm{RDN}$ with radiofrequency ablation has shown to be very promising in improving cardiac function and exercise tolerance for patients with chronic systolic $\mathrm{HF}$, in the present singlecenter, randomized, controlled trial. No adverse effects on the kidneys of patients should also be noted. Thus, this innovative technique can be an effective and safe new therapy for chronic systolic $\mathrm{HF}$, even though more long-term experimental and clinical evidence should be provided in the future studies.

\section{Acknowledgements}

This study was supported by National Natural Science of China (grant No. 81303145); Key Projects of Shanghai Municipal Health Bureau (grant No. 20134003); and Key Medical Discipline Construction Projects of Shanghai Municipal Health Bureau (grant No. ZK2015A147).

\section{Conflict of interest: None declared}

\section{References}

1. Lloyd-Jones D, Adams R, Carnethon M, et al. Heart disease and stroke statistics--2009 update: a report from the American Heart Association Statistics Committee and Stroke Statistics Subcommittee. Circulation. 2009; 119(3): e21-181, doi: 10.1161/CIRCULATIONAHA.108.191261, indexed in Pubmed: 19075105.

2. Dhakal P, Liu K, Kozman H, et al. Renal Denervation in Heart Failure: A New Therapeutic Paradigm. Clin Med Insights Cardiol. 2015; 9(Suppl 1): 101-104, doi: 10.4137/CMC.S18754, indexed in Pubmed: 26157338.

3. Böhm M, Ewen S, Kindermann I, et al. Renal denervation and heart failure. Eur J Heart Fail. 2014; 16(6): 608-613, doi: 10.1002/ ejhf.83.

4. Schlaich MP, Socratous F, Hennebry S, et al. Sympathetic activation in chronic renal failure. J Am Soc Nephrol. 2009; 20(5): 933-939, doi: 10.1681/ASN.2008040402, indexed in Pubmed: 18799718.

5. Patel HC, Dhillon PS, Mahfoud F, et al. The biophysics of renal sympathetic denervation using radiofrequency energy. Clin Res Cardiol. 2014; 103(5): 337-344, doi: 10.1007/s00392-013-0618-6, indexed in Pubmed: 24077678.

6. Renal sympathetic denervation in patients with treatmentresistant hypertension (The Symplicity HTN-2 Trial): a randomised controlled trial. Lancet. 2010; 376(9756): 1903-1909, doi: 10.1016/s0140-6736(10)62039-9.
7. Krum H, Schlaich M, Whitbourn R, et al. Catheter-based renal sympathetic denervation for resistant hypertension: a multicentre safety and proof-of-principle cohort study. Lancet. 2009; 373(9671): 1275-1281, doi: 10.1016/S0140-6736(09)60566-3, indexed in Pubmed: 19332353.

8. Bhatt DL, Kandzari DE, O'Neill WW, et al. SYMPLICITY HTN-3 Investigators. A controlled trial of renal denervation for resistant hypertension. N Engl J Med. 2014; 370(15): 1393-1401, doi: 10.1056/NEJMoa1402670, indexed in Pubmed: 24678939.

9. Abramovitch J, Arviddson B, Dunn K, et al. The radiation tolerance of specific optical fibers for the LHC upgrades. Physics Procedia. 2012; 37: 1630-1643, doi: 10.1016/j.phpro.2012.03.751.

10. Liu Q, Li Z, Zhang Qi, et al. [Effects of renal denervation on left atrial fibrosis in rats with isoproterenol induced chronic heart failure]. Zhonghua Xin Xue Guan Bing Za Zhi. 2015; 43(12): 1040-1045, indexed in Pubmed: 26888837.

11. Xie Y, Liu Q, Xu Y, et al. [Effect of catheter-based renal sympathetic denervation in pigs with rapid pacing induced heart failure]. Zhonghua Xin Xue Guan Bing Za Zhi. 2014; 42(1): 48-52, indexed in Pubmed: 24680270.

12. Davies JE, Manisty CH, Petraco R, et al. First-in-man safety evaluation of renal denervation for chronic systolic heart failure: primary outcome from REACH-Pilot study. Int J Cardiol. 2013; 162(3): 189-192, doi: 10.1016/j.ijcard.2012.09.019, indexed in Pubmed: 23031283.

13. Hasking GJ, Esler MD, Jennings GL, et al. Norepinephrine spillover to plasma in patients with congestive heart failure: evidence of increased overall and cardiorenal sympathetic nervous activity. Circulation. 1986; 73(4): 615-621, indexed in Pubmed: 3948363.

14. Florea VG, Cohn JN. The autonomic nervous system and heart failure. Circ Res. 2014; 114(11): 1815-1826, doi: 10.1161/CIRCRESAHA.114.302589, indexed in Pubmed: 24855204.

15. Kshatriya S, Kozman H, Siddiqui D, et al. The kidney in heart failure: friend or foe? Am J Med Sci. 2012; 344(3): 228-232, doi: 10.1097/MAJ.0b013e318242a631, indexed in Pubmed: 22317900.

16. Tan LH, Li XG, Guo YZ, et al. [Effect of renal sympathetic denervation on left ventricular hypertrophy and inflammatory factors in spontaneously hypertensive rats]. Zhejiang Da Xue Xue Bao Yi Xue Ban. 2013; 42(5): 550-555, indexed in Pubmed: 24167137.

17. Mahfoud F, Urban D, Teller D, et al. Effect of renal denervation on left ventricular mass and function in patients with resistant hypertension: data from a multi-centre cardiovascular magnetic resonance imaging trial. Eur Heart J. 2014; 35(33): 2224-2231, doi: 10.1093/eurheartj/ehu093, indexed in Pubmed: 24603307.

18. Brandt MC, Mahfoud F, Reda S, et al. Renal sympathetic denervation reduces left ventricular hypertrophy and improves cardiac function in patients with resistant hypertension. J Am Coll Cardiol. 2012; 59(10): 901-909, doi: 10.1016/j.jacc.2011.11.034, indexed in Pubmed: 22381425.

19. Patel HC, Rosen SD, Hayward C, et al. Renal denervation in heart failure with preserved ejection fraction (RDT-PEF): a randomized controlled trial. Eur J Heart Fail. 2016; 18(6): 703-712, doi: 10.1002/ejhf.502, indexed in Pubmed: 26990920. 